\title{
Caracterización del comportamiento frente a la corrosión en soldaduras de recargue de aceros inoxidables superdúplex
}

\section{Corrosion characterization in superduplex stainless steel cladding}

\author{
Sebastián Zappa ${ }^{1,2}$, Héctor Pérez ${ }^{3}$, Hernán Svoboda ${ }^{3}$
}

Estela Surian ${ }^{4}$

\author{
${ }^{1}$ Grupo de Investigación en Soldadura - FI-UNLZ CP: 1832, Lomas de Zamora, Buenos Aires, Argentina \\ ${ }^{2}$ CONICET CP: 1425, Ciudad Autónoma de Buenos Aires (CABA), Buenos Aires, Argentina \\ ${ }^{3}$ INTI CP: 1650, San Martín, Buenos Aires, Argentina \\ ${ }^{4}$ Investigadora independiente (CONICET), CP: 1431, Ciudad Autónoma de Buenos Aires (CABA), Buenos Aires, Ar- \\ gentina \\ e-mail: zappasebastian@hotmail.com; hiperez@inti.gob.ar; hsvobod@gmail.com; esurianster@gmail.com
}

\begin{abstract}
RESUMEN
Los aceros inoxidables superdúplex poseen una estructura dual con un 50\% de ferrita y austenita. Con este balance de fases se obtienen las mejores propiedades mecánicas y de resistencia a la corrosión. Gracias a estas características su empleo se encuentra en crecimiento, fundamentalmente, en las industrias del papel, químicas, del gas y del petróleo. La soldadura de recargue, o cladding, está asociada a la deposición de material superficialmente y es utilizada habitualmente tanto para la fabricación como para la reparación de equipos. Las propiedades del recubrimiento dependen de la composición química y de la microestructura, las que a su vez son dependientes de procedimiento de soldadura. Por lo tanto, en soldadura de recargue con aceros inoxidables superdúplex, el control de las fases presentes en la microestructura es esencial para garantizar las propiedades requeridas. El objetivo de este trabajo fue el de estudiar el efecto del calor aportado (alto, medio y bajo) y de la cantidad de capas (una y dos) del depósito de soldadura de acero inoxidable superdúplex sobre la composición química, la microestructura, la dureza y resistencia a la corrosión por picado. Para tal fin, se soldaron seis cupones de recargue mediante el proceso de soldadura por arco eléctrico semiautomático con protección gaseosa: 1 y 2 capas soldados con bajo, medio y alto calor aportado (modificando únicamente la velocidad de soldadura). Sobre los mismos se caracterizó la macro y microestructura, se determinó la dilución geométrica y química, se observó la microestructura mediante microscopía óptica, electrónica de barrido y difracción de rayos $\mathrm{X}$, se determinó la microdureza Vickers y se evaluó la resistencia a la corrosión por picado mediante curvas de polarización.
\end{abstract}

Palabras clave: Soldadura de Recargue, Acero Inoxidable Superdúplex, resistencia a la corrosión.

\section{ABSTRACT}

Superduplex stainless steels have a dual structure constituted approximately by $50 \%$ of ferrite and $50 \%$ of austenite. This fact produces a good combination of mechanical properties and corrosion resistance. Thanks to these features their use is growing, mainly in the industries of paper, chemical, gas and oil. Surfacing welding is used for the manufacture and / or repair of parts and equipment. The properties of the coating depend on its chemical composition and microstructure, which are defined by the dilution with the base metal and the welding process utilized. Therefore, in cladding welding, the control of the present phases in the microstructure is essential to ensure the required properties. The objective of this work was to study the effect of heat input and the amount of layers of superduplex stainless steel deposits on the chemical composition, microstructure, hardness and pitting corrosion resistance. Six cladding coupons were welded by semiautomatic arc welding process: 1 and 2 layers coupons were welded with low, medium and high heat input (modifying only the welding speed). Macro and microstructure was analyzed by optical and scanning electron mi- 
croscopy and X-ray diffraction. The geometrical and chemical dilutions were determined. Also, Vickers microhardness and pitting corrosion resistance were measured.

Keywords: Surfacing Welding, Superduplex Stainless Steel, Corrosion Resistance.

\section{INTRODUCCIÓN}

Las procesos de soldadura por arco eléctrico se utilizan para la unión de piezas (soldadura de unión) y para la protección de superficies sometidas a servicios específicos (soldadura de recargue o cladding). En los últimos años, la soldadura de recargue se ha implementado en numerosas industrias, por una cuestión económica, generando una solución a problemas de ingeniería. Las aplicaciones principales de la soldadura de recargue se encuentran en las industrias del papel, químicas, procesamiento de fertilizantes, procesamiento de alimentos, industria nuclear y en las industrias del gas y del petróleo [1]. El concepto general de la soldadura de recargue se basa en depositar una capa de material con propiedades específicas, fundamentalmente de resistencia a la corrosión o desgaste, sobre un elemento o pieza de acero al carbono o de baja aleación. En forma particular, las características deseables en los recargues de aceros inoxidables a depositar son: buena soldabilidad, buenas propiedades mecánicas y buena resistencia a la corrosión generalizada y localizada. Los aceros inoxidables dúplex poseen todas estas características [2].

Los aceros inoxidables dúplex (DSS) se caracterizan por poseer una estructura dual constituida por ferrita y austenita, con una relación entre ambas fases cercana a 1. Poseen una combinación de buenas propiedades de tracción, tenacidad y resistencia a la corrosión, que dependen de la composición química y de la relación entre las fases mencionada anteriormente. El desarrollo de estos aceros inoxidables ha permitido obtener materiales de alto rendimiento, a partir del aumento de elementos de aleación como el Cr, Mo y N, dando como resultado los aceros inoxidables superdúplex (SDSS) [3]. Las propiedades de los SDSS están estrictamente relacionadas a la composición química, el equilibrio microestructural de ferrita y austenita y a la presencia de fases secundarias. Se ha encontrado que la precipitación de fases tales como nitruros de cromo $\left(\mathrm{Cr}_{2} \mathrm{~N}\right)$, austenita secundaria $\left(\gamma_{2}\right)$ y compuestos intermetálicos como las fases sigma $(\sigma)$ y chi $(\chi)$, tienen una gran influencia sobre las propiedades y el rendimiento de estos aceros [4]. Debido a su alta resistencia a la corrosión y propiedades mecánicas mejoradas se utilizan ampliamente en plantas petroquímicas tales como instalaciones en las plataformas petrolíferas y equipos modernos en procesos off-shore. Tubos, bombas, recipientes a presión, separadores e intercambiadores de calor son algunos ejemplos de estas aplicaciones [5].

El procedimiento de soldadura tiene un rol relevante en las características de los depósitos obtenidos, ya que se puede modificar el equilibrio entre fases y generar la precipitación de fases secundarias obteniendo una pérdida importante de las propiedades. En términos generales, en soldaduras de SDSS, altas velocidades de enfriamiento, pueden dar como resultado una excesiva cantidad de ferrita y la precipitación de nitruros. Por otro lado, una baja velocidad de enfriamiento puede generar la precipitación de fases intermetálicas [6].

Además, la composición química, la microestructura y las diferentes propiedades de los materiales de recargue son dependientes del grado de dilución del metal aportado y el metal base fundido, sobre el cuál ése se deposita y se mezcla. Esta dilución es también afectada por el procedimiento de soldadura. La dilución reduce la concentración de elementos de aleación y aumenta el contenido de carbono en la capa de recargue (dado que el material base generalmente posee mayor contenido de este elemento que un acero dúplex), lo que disminuye las propiedades de resistencia a la corrosión y causa otros problemas metalúrgicos. Un grado de dilución no controlado en un recargue, puede producir un desequilibrio de las fases microestructurales presentes (50\% de ferrita y $50 \%$ de austenita), que caracterizan a estos aceros [1,2].

El objetivo de este trabajo fue estudiar el efecto del calor aportado y de la cantidad de capas del depósito de soldadura de acero inoxidable superdúplex sobre la composición química, el grado de dilución, la microestructura, la microdureza y la resistencia a la corrosión por picado.

\section{MATERIALES Y MÉTODOS}

Se soldaron seis cupones de soldadura de recargue, sobre una chapa de acero comercial de bajo carbono mediante el proceso de soldadura semiautomático con protección gaseosa (GMAW). A fin de variar el aporte térmico (HI), se emplearon, en todos los casos, los mismos parámetros eléctricos modificando únicamente la velocidad de soldadura, de manera de generar diferentes velocidades de enfriamiento. Los parámetros de soldadura se eligieron garantizando un arco eléctrico estable y se varió la velocidad de soldadura con el propósito de poder comparar situaciones extremas de aporte térmico. El consumible empleado fue un alambre macizo de 1,2 mm de diámetro que deposita un SDSS. Las soldaduras fueron efectuadas en posición plana, bajo mano y realizadas con un dispositivo mecanizado. El gas de protección fue $\mathrm{Ar}+20 \% \mathrm{CO} 2$, con un caudal de 
$18 \mathrm{~L} / \mathrm{min}$ y la distancia entre el pico de contacto y la pieza, en todos los casos, fue de $20 \mathrm{~mm}$. Asimismo, se analizó el efecto de la cantidad de capas, realizándose probetas con una y dos capas. La configuración de los cupones de recargue fue cinco cordones en la primer capa y cuatro en la segunda. Las chapas utilizadas como base fueron de acero comercial de bajo contenido de C de 250 × 75 × 9,5 mm. La Tabla 1 muestra la identificación de las probetas, los parámetros de soldadura promedio y el calor aportado en cada caso. Siendo: T: tensión, C: corriente; VS: velocidad de soldadura y CA: calor aportado = T x C / VS x 100 .

Tabla 1: Identificación de las probetas y parámetros de soldadura.

\begin{tabular}{l|l|l|l|l}
\hline CUPÓN & $\mathbf{T}(\mathbf{V})$ & $\mathbf{C}(\mathbf{A})$ & VS $(\mathbf{m m} / \mathbf{s})$ & $\mathbf{C} \mathbf{A}(\mathbf{k J} / \mathbf{m m})$ \\
\hline S1B & 28 & 203 & 9,4 & 0,6 \\
\hline S1M & 32 & 208 & 5,5 & 1,2 \\
\hline S1A & 27 & 203 & 3,1 & 1,8 \\
\hline S2B & 28 & 206 & 9,7 & 0,6 \\
\hline S2M & 31 & 210 & 5,6 & 1,2 \\
\hline S2A & 27 & 206 & 3,1 & 1,8 \\
\hline
\end{tabular}

La Tabla 2 muestra la composición química de la chapa base y del electrodo consumible reportado por el fabricante [7]. La Figura 1 muestra fotografías de los equipos de soldadura empleados (fuente GMAW; cabezal GMAW; consumible SDSS; tubo de gas de protección; torcha; dispositivo para automatizar las soldaduras a velocidad constante).

Tabla 2: Composición química de la chapa base y del consumible utilizado.

\begin{tabular}{l|l|l|l|l|l|l|l|l}
\hline & C (\%) & Si (\%) & Mn (\%) & Cr (\%) & Ni (\%) & Mo (\%) & Cu (\%) & W (\%) \\
\hline Chapa & 0,10 & 0,20 & 0,58 & - & - & - & - & - \\
\hline Consumible & 0,02 & 0,30 & 0,70 & 25,2 & 9,2 & 3,6 & 0,6 & 0,62 \\
\hline
\end{tabular}

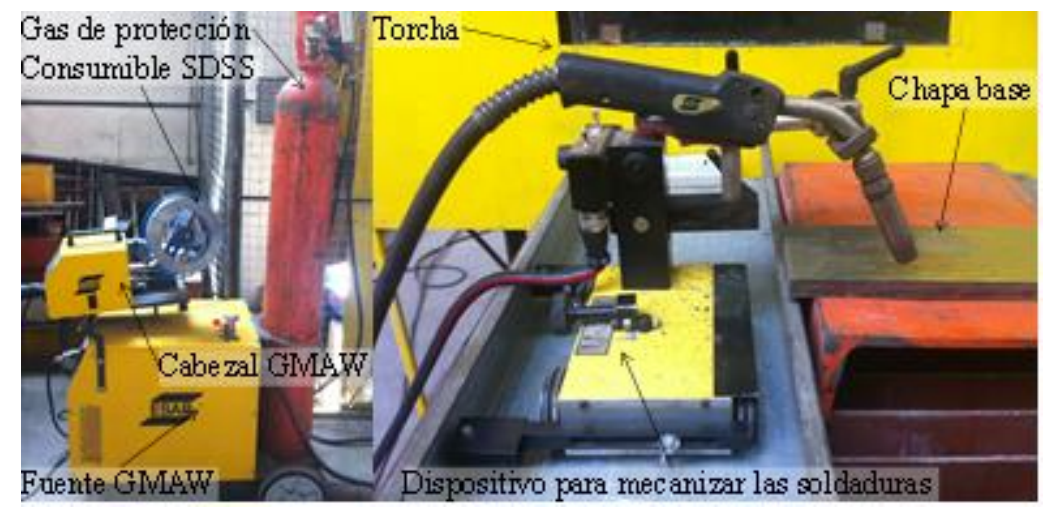

Figura 1: Geometría del recargue y dilución geométrica.

Una vez soldados los cupones, se realizó una inspección visual y se tomaron fotos superficiales de los mismos. Para la caracterización macroestructural y microestructural se extrajeron cortes transversales. Dichos cortes fueron preparados metalográficamente (desbaste con papeles abrasivos y pulidos con pasta de diamante). El revelado de la estructura se llevó a cabo por medio de un ataque electrolítico con una solución de 40 gr $\mathrm{NaOH}+$ agua con una tensión de $3 \mathrm{~V}$ durante un tiempo no mayor a $10 \mathrm{~s}$. En dichas muestras, se determinó la geometría (ancho, alto y penetración) y la dilución geométrica de los recargues. Esto es la relación entre el área del metal base fundido (B) respecto el área total del metal de soldadura (A + B) [2], como se muestra en la Figura 2. En la superficie de cada recargue se midió la composición química mediante espectrometría de emisión óptica y se determinó la dilución química, siendo ésta la relación entre la composición química medida experimentalmente y la reportada por el fabricante del consumible sin efecto de dilución (metal de aporte puro). Se caracterizó la microestructura mediante microscopía óptica y microscopía electrónica de barrido. Además, para poder alcanzar mayor nivel de identificación de fases se realizaron ensayos de difracción de rayos $\mathrm{X}$ con radiación de $\mathrm{Cu}\left(\lambda=1,5408 \AA\right.$ ) en un rango de 30 a $90^{\circ}$ ( 2 tita) con un paso y velocidad de barrido de $0,05^{\circ}$ y $0,5^{\circ} / \mathrm{min}$, respectivamente. Los espectros obtenidos fueron procesa- 
dos en software para una correcta identificación de fases. En algunas muestras microestructurales, se utilizó la técnica de Espectrometría de rayos X dispersiva en energía (EDS) para completar la identificación de ciertos precipitados.

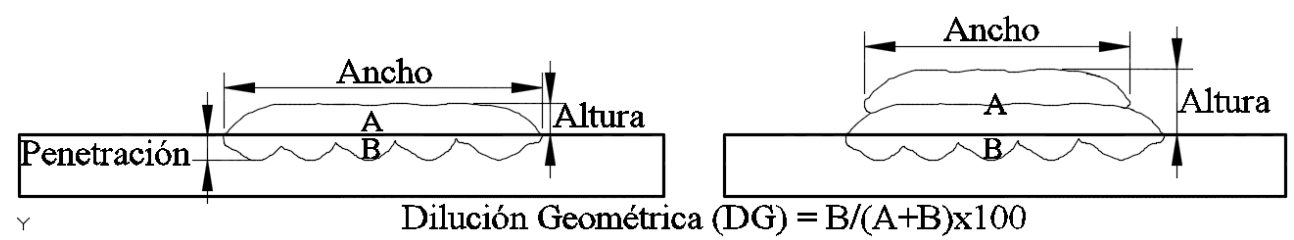

Figura 2: Geometría del recargue y dilución geométrica.

Se realizó la cuantificación microestructural de ferrita y austenita por medio de metalografía cuantitativa con un software de análisis de imágenes en 10 campos a 500x, ponderando el porcentaje de fases oscuras (ferrita) respecto de las claras (austenita). Se tomaron al menos cinco valores de dureza Vickers con $1 \mathrm{~kg}$ de carga reportándose los valores promedio para cada condición.

A fin de caracterizar el comportamiento frente a la corrosión de los cupones soldados, se realizó un estudio electroquímico en la superficie libre (cara superficial) de los recargues. La zona de medición, en todos los casos, fue ubicada sobre los últimos cordones soldados para obtener resultados comparables. Para efectuar estas mediciones se utilizó un potenciostato/galvanostato marca Autolab, modelo 302N, y una celda electroquímica de apoyo de $3 \mathrm{~mm}$ de diámetro. Como contra-electrodo se utilizó un alambre de platino (Pt) y un electrodo de referencia de plata/cloruro de plata $(\mathrm{Ag} / \mathrm{AgCl})$. Dicho estudio consistió en la medición del potencial de corrosión $\left(\mathrm{E}_{\mathrm{CORR}}\right)$ durante $200 \mathrm{~s}$, seguido por una curva potenciodinámica cíclica con una velocidad de barrido de $1 \mathrm{mV} / \mathrm{s}$. El potencial de partida fue $-100 \mathrm{mV}$ vs Ecorr, hasta alcanzar una densidad de corriente de $1.10^{-4} \mathrm{~A} / \mathrm{cm} 2$, valor denominado Ilim. A partir del potencial correspondiente a esta corriente se invirtió el sentido de avance, polarizando catódicamente hasta el potencial de partida. Ambas medidas se realizaron en condiciones de aireación natural, sin eliminación del oxígeno. Con dicha técnica se obtiene el potencial de picado $\left(\mathrm{E}_{\mathrm{PIC}}\right)$, la densidad de corriente máxima (Imax) y el potencial crítico de picado o de repasivación $\left(\mathrm{E}_{\mathrm{REP}}\right)$. Un mínimo de 5 mediciones electroquímicas fueron realizadas sobre cada cupón de soldadura reportándose los valores promedios.

\section{RESULTADOS}

La Figura 3 muestra el aspecto superficial de los recargues obtenidos.
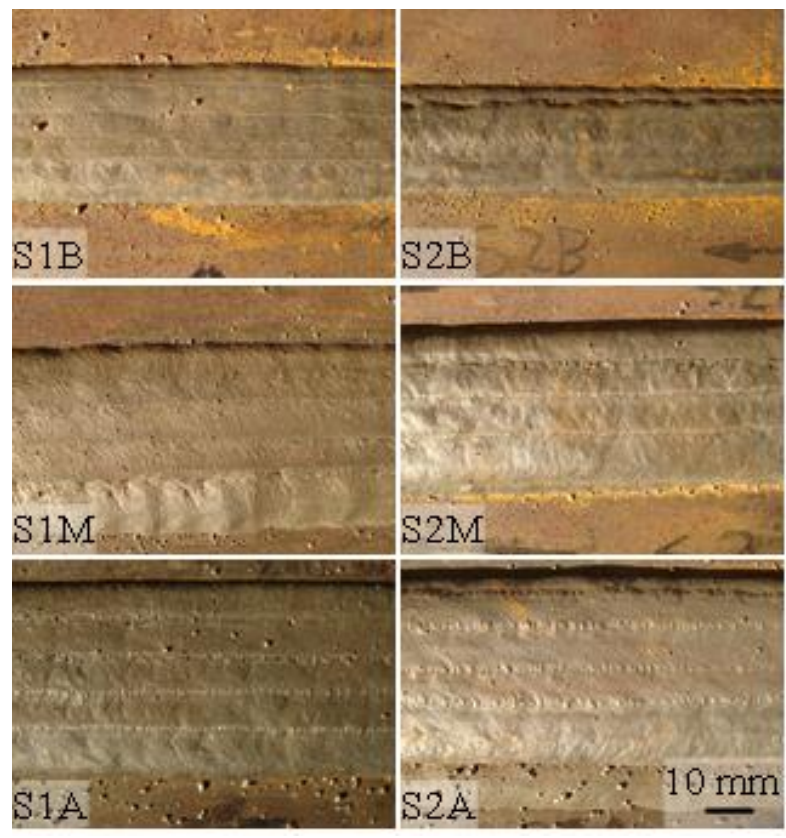

Figura 3: Aspecto superficial de los cordones. 
Se puede observar, en términos generales, bajos niveles de salpicaduras y de escoria. No se observaron discontinuidades mediante inspección visual en los diferentes cupones. Además, los recargues presentaron una superficie plana con una buena terminación.

En la Figura 4 se muestran los cortes transversales de las distintas probetas soldadas. En dichas imágenes se pueden observar las diferentes zonas que caracterizan a los cupones de recargue: Chapa base, zona afectada por el calor y el metal de soldadura conformando el recargue (con una o dos capas). Además, en el cupón S2A, se observa una discontinuidad parcial entre la primera y segunda capa del recargue, asociada a una falta de fusión entre cordones generado por una mala posición de la torcha al realizar las soldaduras.

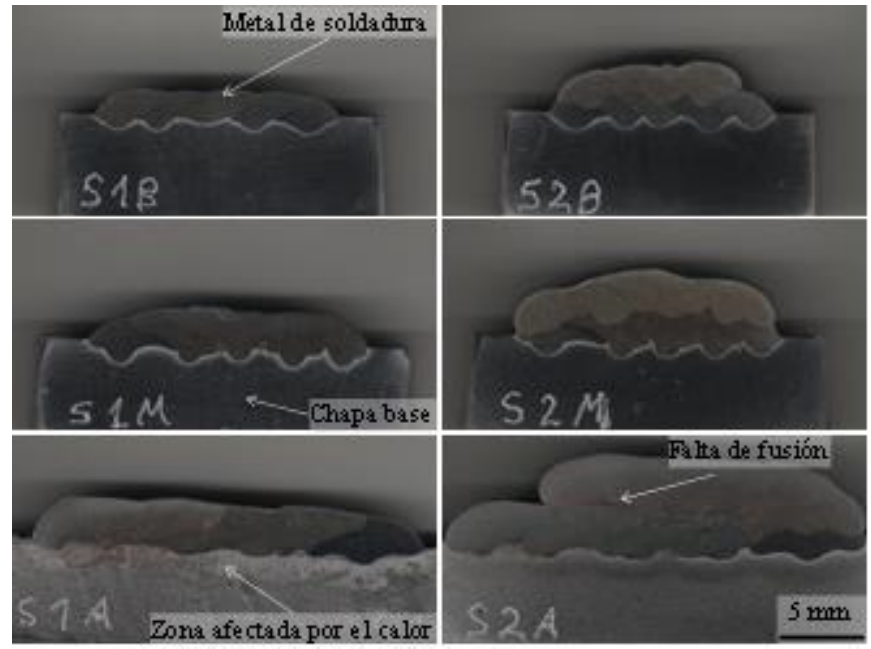

Figura 4: Macrografías de cortes transversales de los recargues.

La Tabla 3 muestra las dimensiones, la composición química y la dilución geométrica y química, de cada uno de los recargues. Siendo: AN: ancho, AL: alto y P: penetración, DG: dilución geométrica y química DQ: dilución química.

Tabla 3: Características geométricas, composición química y valores de dilución calculados de los recargues soldados.

\begin{tabular}{l|l|l|l|l|l|l|l|l|l|l|l}
\hline CUPÓN & AN (mm) & AL (mm) & P (mm) & DG (\%) & Mn (\%) & Cr (\%) & Ni (\%) & Mo (\%) & Cu (\%) & W (\%) & DQ (\%) \\
\hline S1B & 28,8 & 3,1 & 1,7 & $\mathbf{3 2}$ & 0,7 & 16,7 & 6,4 & 2,6 & 0,24 & 0,38 & $\mathbf{3 2}$ \\
\hline S1M & 33,1 & 4,2 & 2,5 & $\mathbf{3 0}$ & 0,8 & 19,1 & 7,2 & 2,9 & 0,37 & 0,40 & $\mathbf{2 3}$ \\
\hline S1A & 46,3 & 5,3 & 2,1 & $\mathbf{2 8}$ & 0,9 & 24,1 & 9,1 & 3,7 & 0,43 & 0,53 & $\mathbf{3}$ \\
\hline S2B & 26,7 & 6,1 & 1,9 & $\mathbf{2 2}$ & 0,8 & 21,9 & 8,4 & 3,3 & 0,40 & 0,49 & $\mathbf{1 1}$ \\
\hline S2M & 31,2 & 7,9 & 2,4 & $\mathbf{1 9}$ & 0,8 & 22,8 & 8,8 & 3,5 & 0,42 & 0,47 & $\mathbf{7}$ \\
\hline S2A & 49,7 & 10,4 & 2,2 & $\mathbf{1 7}$ & 0,9 & 25,1 & 9,5 & 3,8 & 0,52 & 0,51 & $\mathbf{1}$ \\
\hline
\end{tabular}

De acuerdo a los valores reportados en la tabla anterior, se puede observar que las dimensiones del recargue se vieron afectadas por los parámetros de proceso empleados. El ancho y la altura de los recargues aumentaron al aumentar el calor aportado, tanto para los cupones soldados con una capa, como para los soldados con dos capas. Sin embargo, la penetración no tuvo ese comportamiento. Es decir, la penetración aumentó hasta 2,5 mm para una capa y 2,4 mm para dos capas, y luego disminuyó al aumentar el calor aportado.

Además, realizando el cociente del área transversal de la zona fundida de la chapa base respecto al área total del recargue $(B / A+B$, Figura 2) se puede observar que la dilución geométrica fue influenciada por las diferentes condiciones estudiadas. En este sentido, la dilución geométrica disminuyó de 32 a $28 \%$ para los recargues de una capa y de 22 a $17 \%$ para los de dos capas, al aumentar el calor aportado.

Respecto a los valores de composición química determinados por espectrometría de emisión óptica sobre la superficie libre de los recargues, se puede observar que el nivel de aleación crece monótonamente al aumentar el calor aportado, tanto en los cupones soldados con una capa, como así también en los soldados con dos capas. Sin embargo, se destaca que la tasa de crecimiento de aleación para ambas condiciones fue diferente. Es decir, para los recargues de una capa se evidencia un mayor crecimiento de los diferentes ele- 
mentos de aleación, mientras que para los recargues de dos capas el crecimiento es menor. Finalmente, al igual que en el caso de la dilución geométrica, la dilución química disminuyó al aumentar el calor aportado de 32 a $3 \%$ para una capa y de 11 a $1 \%$ para dos capas.

La Figura 5 y 6 muestran la microestructura superficial obtenida para los diferentes recargues soldados por microscopía óptica y electrónica de barrido, respectivamente. En dichas micrografías se puede observar una estructura constituida por dos fases: ferrita y austenita. Además, en todos los casos, se puede apreciar un patrón de solidificación dendrítico. Finalmente, se puede identificar la presencia de algunas inclusiones observadas mediante Difracción de Rayos X y caracterizadas por con Espectrometría de Rayos X Dispersiva en Energía (EDS).

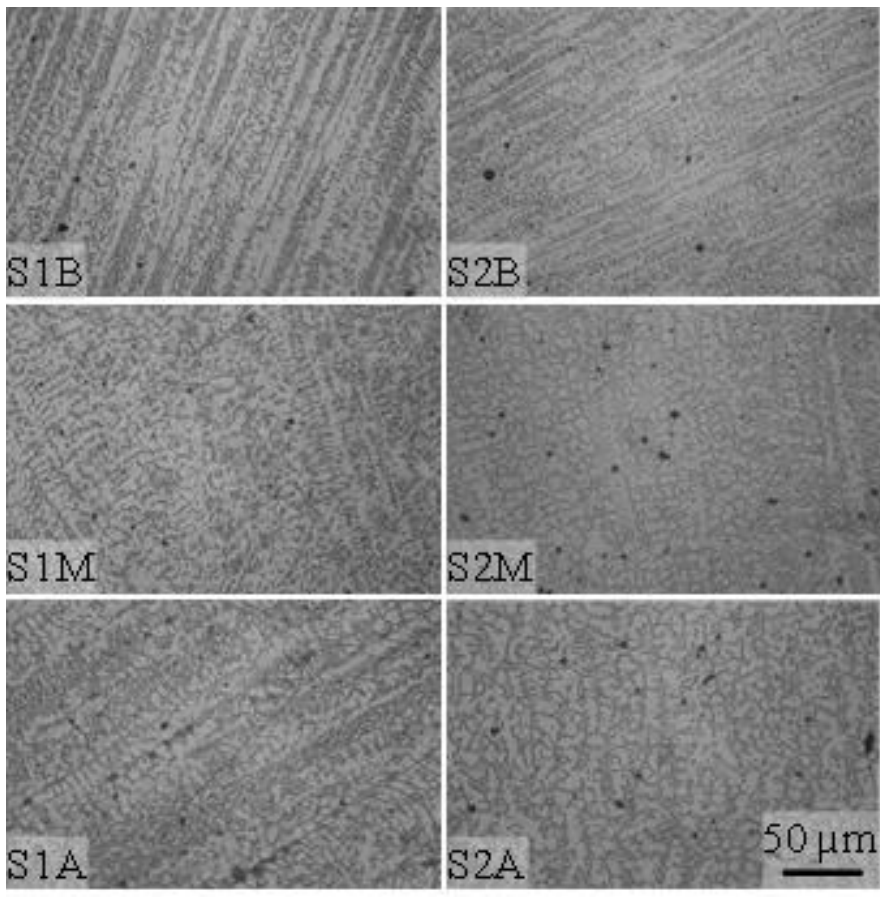

Figura 5: Microestructuras superficiales de los recargues: microscopía óptica.
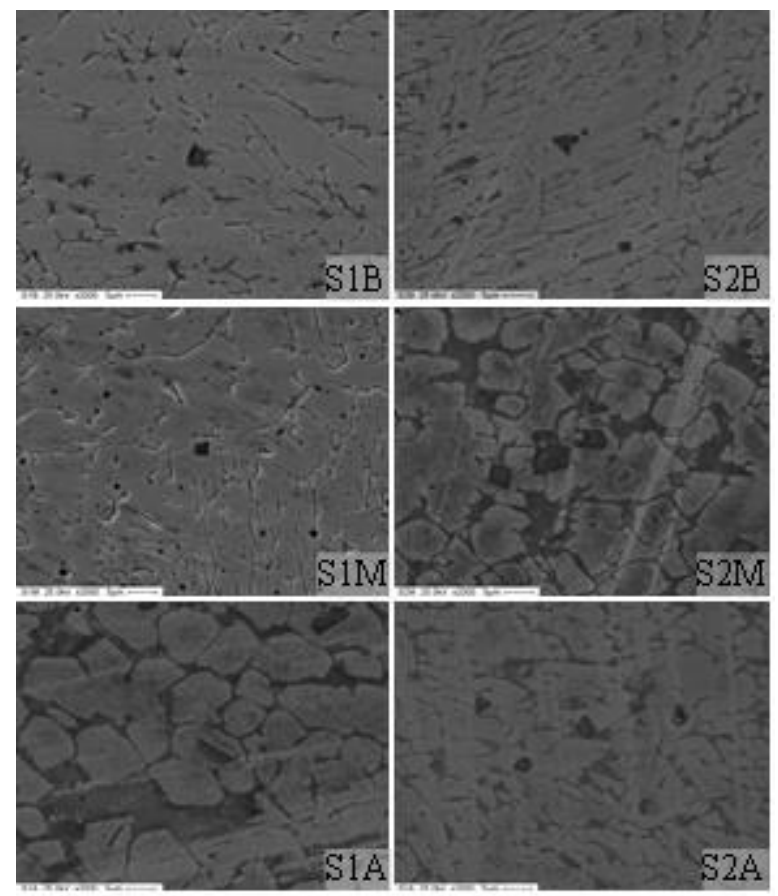

Figura 6: Microestructuras superficiales de los recargues: microscopía electrónica de barrido. 
La Figura 7 muestra un detalle de la muestra S2B donde se midió la composición química sobre un precipitado identificado como un óxido, fundamentalmente de $\mathrm{Cr}$, silicio y aluminio. La Figura 8 muestra los espectros de Difracción de Rayos X. En dichos espectros se puede identificar los picos de difracción correspondientes a la ferrita y a la austenita. Al aumentar el calor aportado los picos correspondientes a la ferrita y a la austenita, aumentan y disminuyen respectivamente, para ambas capas indicando un aumento del contenido de ferrita y una disminución del contenido de austenita. Sin embargo, dicha evolución es más significativa en los recargues de una capa. Además, se observan pequeños picos de difracción ubicados en los ángulos 2 tita $=37$ y $77^{\circ}$, correspondientes a óxidos de Al-Si, caracterizados mediante EDS.

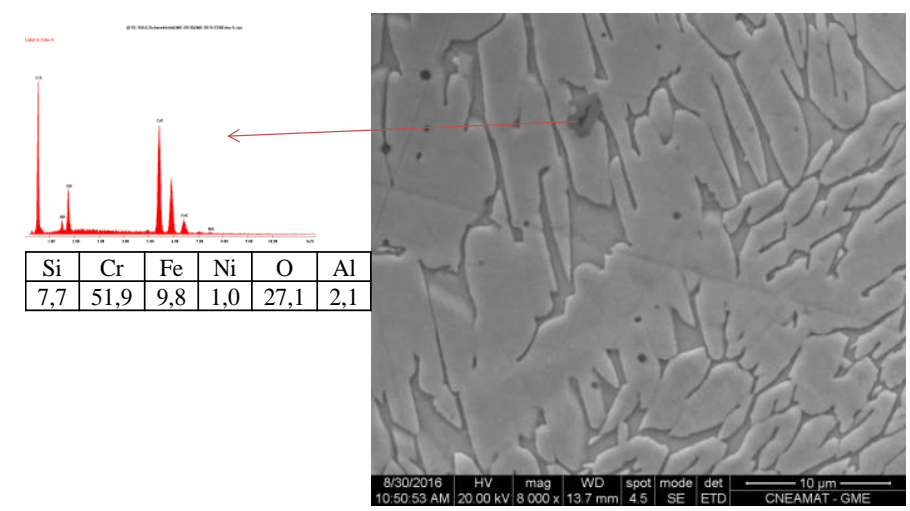

Figura 7: Muestra S2B, caracterización de un precipitado.

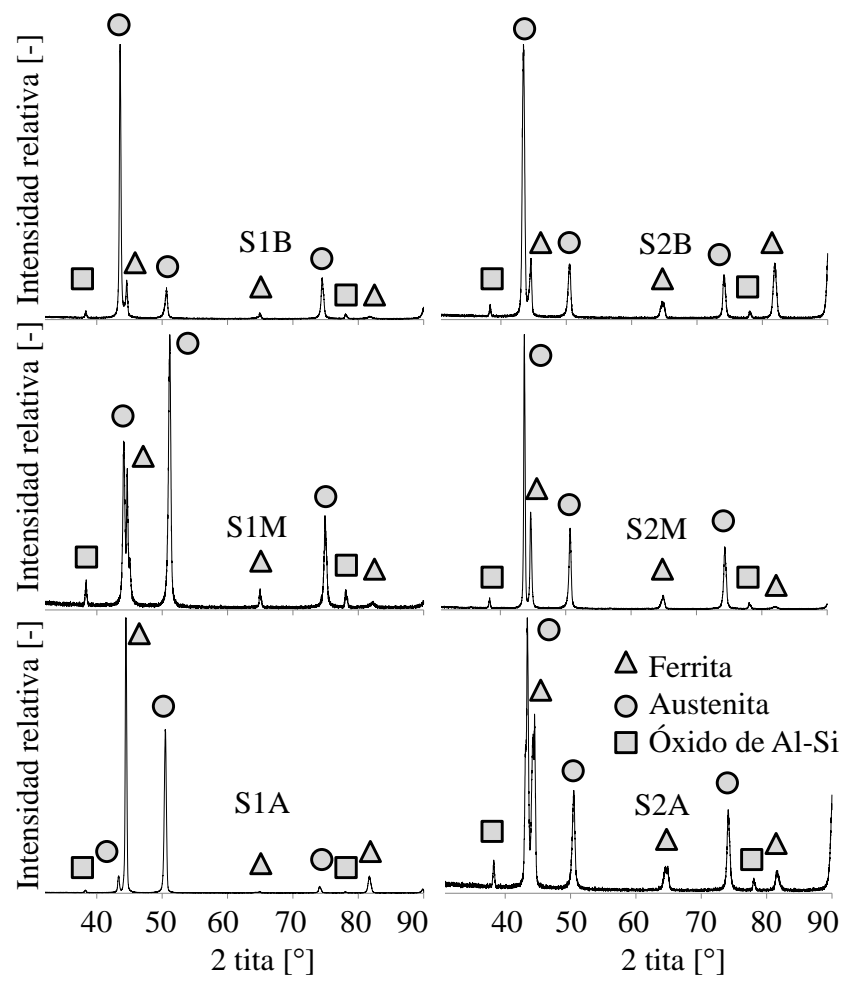

Figura 8: Espectros de Difracción de Rayos X.

La Tabla 4 muestra el porcentaje de fases (ferrita y austenita), los valores promedios de dureza, el potencial de corrosión $\left(\mathrm{E}_{\mathrm{CORR}}\right)$, la corriente de corrosión $\left(\mathrm{I}_{\mathrm{CORR}}\right)$, el potencial de picado ( $\left.\mathrm{E}_{\mathrm{PIC}}\right)$ y el potencial de repasivación $\left(\mathrm{E}_{\mathrm{REP}}\right)$ determinados en la superficie de los recargues. La Figura 9 muestra, a modo de ejemplo, curvas de polarización representativas de las diferentes condiciones soldadas.

En dicha tabla se puede observar que el equilibrio microestructural fue afectado por las variables estudiadas. Los recargues soldados con dos capas presentaron una microestructura más cercana al balance ideal, respecto a los recargues soldados con una capa. Por otro lado, en ambos casos, al aumentar el calor aportado el contenido de ferrita aumentó de 22 a $36 \%$ y de 32 a $39 \%$ para los cupones soldados con una y dos capas, 
respectivamente. Sin embargo, para la condición S1A se obtuvieron resultados similares a los de los recargues soldados con dos capas.

Tabla 4: Características geométricas de los recargues soldados.

\begin{tabular}{|c|c|c|c|c|c|c|c|}
\hline CUPÓN & FERRITA (\%) & AUSTENITA (\%) & DUREZA $\left(\mathrm{H}_{\mathrm{v} 1}\right)$ & $E_{\text {CORR }}(\mathrm{V})$ & $I_{\text {CORR }}\left(\mathrm{mA} / \mathrm{cm}^{2}\right)$ & $E_{\text {PIC }}(\mathrm{V})$ & $E_{\text {REP }}(V)$ \\
\hline S1B & 22 & 78 & 245 & $-0,087$ & $1,8 \mathrm{E}-06$ & 1,20 & 1,19 \\
\hline $\mathrm{S} 1 \mathrm{M}$ & 24 & 76 & 275 & $-0,089$ & $3,4 \mathrm{E}-07$ & 1,13 & 1,09 \\
\hline S1A & 36 & 64 & 285 & $-0,190$ & $2,0 \mathrm{E}-06$ & 1,14 & 1,14 \\
\hline S2B & 32 & 68 & 274 & 0,048 & $3,3 \mathrm{E}-08$ & 1,18 & 1,14 \\
\hline $\mathrm{S} 2 \mathrm{M}$ & 36 & 64 & 278 & 0,107 & $5,9 \mathrm{E}-08$ & 1,19 & 1,15 \\
\hline $\mathrm{S} 2 \mathrm{~A}$ & 39 & 61 & 283 & 0,064 & $2,9 \mathrm{E}-08$ & 1,10 & 1,05 \\
\hline
\end{tabular}

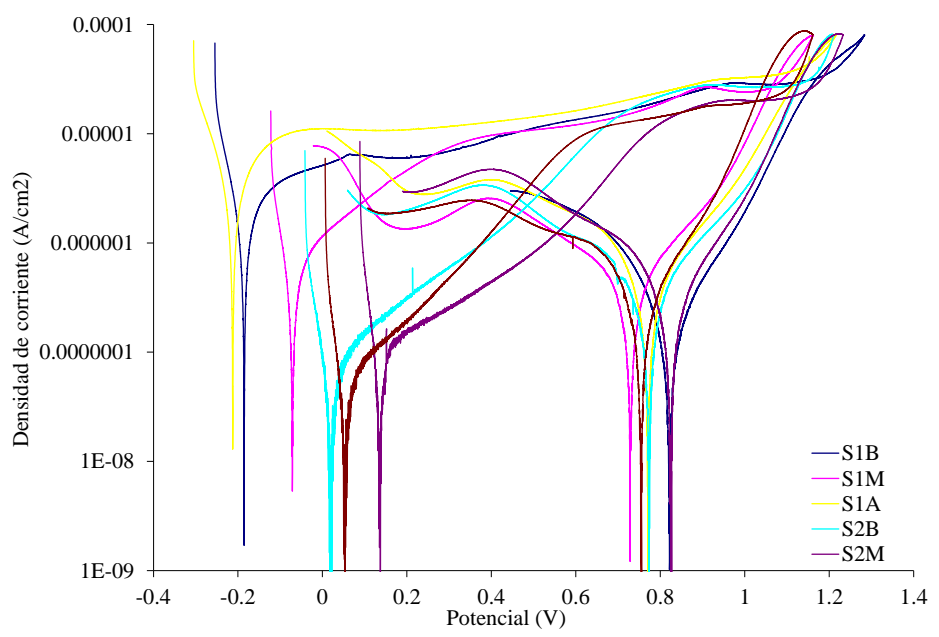

Figura 9: Curvas de polarización anódica.

Finalmente, de acuerdo a los valores promedio de los ensayos de corrosión reportados en la Tabla 4 no se encontraron grandes diferencias en los potenciales característicos que definen la ruptura $\left(\mathrm{E}_{\mathrm{PIC}}\right)$ y nueva generación $\left(\mathrm{E}_{\mathrm{REP}}\right)$ de la película pasiva (corrosión por picado) para las diferentes condiciones analizadas. Sin embargo, se pudo observar que hubo una fuerte incidencia respecto a la corriente de corrosión que caracteriza a la velocidad de disolución de la película pasiva (corrosión generalizada).

Respecto a la dureza, si bien no se encontraron grandes variaciones, se observaron comportamientos definidos. Los valores promedio de dureza fueron dependientes de la condición de soldadura. Al aumentar el calor aportado la dureza aumento de 245 a 285 y de 274 a $283 \mathrm{HV}_{1}$, para los recargues soldados con una y dos capas, respectivamente.

\section{DISCUSIÓN}

Algunos autores [8] comentan que los parámetros dimensionales (ancho, alto y penetración) de los cordones soldados son inversamente proporcionales a la velocidad de soldadura, a parámetros electricos constantes. Es decir, al aumentar la velocidad de soldadura disminuye el calor aportado por unidad de longitud, generando una disminución en el ancho y la altura y una menor fusión del metal base, produciendo una menor penetración. Otros autores [2], establecen que a mayor velocidad de soldadura el ancho y la altura del cordón disminuye, pero la penetración aumenta. La disminución en el ancho y la altura del cordón pueden ser atribuidas a la disminución del calor aportado por unidad de longitud de soldadura, sabiendo que a mayor velocidad de soldadura, menor cantidad de material por unidad de tiempo se deposita. Con una velocidad de soldadura baja, el arco eléctrico es casi vertical y, en este caso, la pileta líquida amortigua el efecto de arco y evita una penetración mayor [1,2], efecto evidenciado en el presente trabajo por medio de la penetración. Asimismo, se puede ver que la segunda capa prácticamente duplica la altura del depósito.

La dilución geométrica general del recargue osciló entre un 32 y un $17 \%$ para las distintas condiciones de soldadura. En este sentido, este hecho es un dato relevante, dada la importancia de la composición 
química en la formación de la microestructura [1]. El porcentaje de dilución del metal base en la pileta líquida disminuyó con el aumento del calor aportado. Para una capa osciló entre 32 y $28 \%$, mientras que con dos capas se ubicó entre un 22 y 17\%. Para una misma cantidad de capas, es interesante notar que al aumentar el calor aportado de 0,6 a 1,8 kJ/mm no generó grandes variaciones en la dilución geométrica de los recargues, siendo ésta de un 30 y $20 \%$ aproximadamente para una y dos capas, respectivamente.

La composición química superficial de los recargues experimentó modificaciones en función a las variables estudiadas (calor aportado y cantidad de capas). Dichas variaciones fueron más evidentes en los cupones soldados con una capa respecto a los soldados con dos capas. Es decir, para los cupones soldados con una capa, al aumentar el calor aportado, el contenido de aleación aumentó más que lo observado en las probetas de dos capas. El aumento del nivel de aleación variando las condiciones del procedimiento de soldadura está fuertemente relacionado con el nivel de dilución discutido anteriormente. Al aumentar el calor aportado, cambia la geometría del metal depositado y del metal base fundido, disminuyendo el grado de dilución y aumentando el nivel de aleación.

Los valores determinados en la dilución química responden a lo observado experimentalmente en la composición química. Los recargues soldados con dos capas presentaron menores valores de dilución, respecto a los recargues de una capa.

En todos los casos, la microestructura estuvo constituida principalmente por dos fases: ferrita y austenita. En este sentido, el contenido de austenita fue mayor que el de ferrita para todos los recargues. Sin embargo, se pudo observar que al aumentar el calor aportado y al pasar de una a dos capas el contenido de ferrita aumentó. El calor aportado es una medida relativa de la energía transferida por unidad de longitud soldada. Éste es un factor importante porque modifica la velocidad de enfriamiento, la cual afecta la evolución microestructural y las propiedades finales del cordón soldado. Según la literatura [1] a menor calor aportado, mayor contenido de ferrita en la microestructura. Esto está asociado a que al disminuir el calor aportado aumenta la velocidad de enfriamiento y el tiempo de transformación de ferrita en austenita es reducido, dando como resultado un mayor contenido de ferrita en la microestructura. Además, con mayor aporte térmico, la pileta líquida permanece más tiempo en este estado, posibilitándose una mayor oxidación de los elementos de bajo potencial de oxidación, que pasan como óxidos a la escoria o al medio, disminuyendo así su contenido en el metal depositado, entre ellos el $\mathrm{Cr}$, uno de los más oxidables y el principal elemento ferritizante [9]. Por otro lado, la composición química del depósito es fuertemente dependiente de la dilución obtenida. El control de la dilución es muy importante durante la soldadura, fundamentalmente en recubrimientos, donde se buscan bajos niveles de dilución. Cuando la dilución es baja, la composición química del recubrimiento es cercana a la composición química del consumible y las propiedades finales del recubrimiento son las deseadas [1]. La literatura [10] menciona que al aumentar los niveles de dilución el contenido de ferrita en la microestructura disminuye. Un aumento de la dilución aumenta el contenido de $\mathrm{C}$ y reduce los contenidos de $\mathrm{Cr}$ y Ni del recubrimiento, aumentando el contenido de austenita en la microestructura. Los cambios en la dilución gobiernan la composición química del recubrimiento. Un aumento en la dilución reduce el contenido de elementos estabilizadores de la ferrita (alfágenos) y también reduce, pero de manera moderada, los elementos estabilizadores de la austenita (gammágenos), dando como resultado una disminución del contenido de ferrita [1, 10]. De acuerdo a lo observado mediante microscopía óptica, electrónica de barrido, difracción de rayos X y a lo discutido anteriormente, se presentan dos efectos opuestos. Por un lado, una disminución de la velocidad de soldadura genera un mayor tiempo de transformación de ferrita en austenita dando como resultado un menor contenido de ferrita en la microestructura [11]. Por otro lado, al disminuir la velocidad de soldadura disminuye la dilución con la chapa base, dando como resultado un mayor contenido de ferrita [1, 10]. En este sentido, a partir de lo observado en las diferentes condiciones analizadas, la disminución de la dilución con la velocidad de soldadura controla el balance estructural, resultando en un aumento del contenido de ferrita.

El calor aportado, modificado por la velocidad de soldadura, influyó en el balance microestructural de los depósitos, al igual que la cantidad de capas asociados al porcentaje de dilución y a la velocidad de enfriamiento discutido anteriormente. Además, considerando los resultados de la Tabla 4, en donde se muestran los valores de dureza para las distintas condiciones de recargue, se observa que, si bien no se encontraron grandes variaciones $\left(40 \mathrm{HV}_{1}\right)$, a menor velocidad de soldadura (mayor calor aportado) mayor fue la dureza. Esto podría estar asociado a los mayores contenidos de ferrita medidos en la microestructura para estas condiciones, ya que la ferrita presenta mayor dureza respecto a la austenita [12].

La capacidad de protección de la película se ve grandemente afectada por efecto de la dilución. Las corrientes de corrosión aumentan en más de un orden de magnitud entre los recubrimientos formados por dos capas (30-80 nA/cm2) respecto a los de una capa (400-2000 nA/cm), llevándolos por encima de los límites considerados como corrientes de pasividad. De igual manera, los potenciales de corrosión de los recubrimientos de simple capa, se ven disminuidos con respecto a los de doble, lo que indica su mayor susceptibilidad a 
la corrosión. No se aprecia un efecto claro del calor aportado en la resistencia de la película.

Respecto al comportamiento frente a la corrosión localizada, no se observa una gran diferencia de susceptibilidad de los recargues en las diferentes condiciones de soldadura en la solución de ensayo utilizada, con potenciales de picado en el entorno de $1,1 \mathrm{~V}-1,2 \mathrm{~V}$. Se observa una tendencia a disminuir levemente el potencial de picado y repasivación al aumentar el calor aportado tanto en una como en dos capas.

\section{CONCLUSIONES}

El aumento del aporte térmico, al disminuir la velocidad de soldadura, en los recargues de SDSS generó el aumento del ancho y altura de los cordones depositados. Sin embargo, no se midieron grandes diferencias en la penetración, generando una menor dilución y aumentando el contenido de ferrita y la dureza del depósito.

Por otro lado, la segunda capa de recargue respecto a una simple capa, generó menores valores de dilución, acercándose al balance de fases buscado. En estos casos la dureza fue superior que para una capa.

No se encontraron diferencias en los potenciales de picado ni de repasivación al aumentar el calor aportado y la cantidad de capas del recargue. Sin embargo, se pudo observar que hubo una fuerte incidencia de la corriente de corrosión en los recargues de una capa respecto a los de dos capas.

El equilibrio microestructural en recargues con SDSS está controlado principalmente por el grado de dilución y en menor medida por la velocidad de enfriamiento. El hecho que la susceptibilidad a la corrosión por picado se vio afectada por la velocidad de enfriamiento y la cantidad de capas es un dato relevante ya que se pueden lograr recubrimientos por soldadura depositando material a la mayor velocidad posible y en la menor cantidad de capas posible.

\section{AGRADECIMIENTOS}

Los autores agradecen a Daniel Betz de BOHELER Argentina por la provisión del consumible utilizado, a Ricardo Flores de AIR LIQUIDE Argentina por la donación de los gases de soldadura, a Eduardo Asta de ESAB-CONARCO Argentina por los análisis químicos, a Mercedes Pianetti del Laboratorio de Microscopía Electrónica del INTI - MECÁNICA por las imágenes SEM y a la UNLZ por el aporte financiero.

\section{BIBLIOGRAFÍA}

[1] KANNAN, T. and MURUGAN, N., "Prediction of Ferrite Number of duplex stainless steel clad metals using RSM", Welding Journal, v. 85, n. 91, pp 91s-100s, 2006.

[2] KANNAN, T. and MURUGAN, N., "Effect of flux cored arc welding process parameters on duplex stainless steel clad quality", Journal of Materials Processing Technology, v. 176, n. 1-3, pp 230-239, 2006.

[3] KARLSSON, L. and ARCINI, H., "Low energy input and high dilution welding of duplex stainless steels", In: International Institute of Welding Conference, Doc. No. IX-2356r1-11, pp 1-11, 2011.

[4] EGHLIMI, A., SHAMANIAN, M., RAEISSI, K., "Effect of current type on microstructure and corrosion resistance of super duplex stainless steel cladding produced by the gas tungsten arc welding process", Surface and Coatings Technology, v. 244, pp 45-51, 2014.

[5] PARDAL, J. M., TAVARES, S. S. M., CINDRA FONSESCA, M., et al., "Influence of the grain size on deleterious phase precipitation in superduplex stainless steel UNS S32750", Materials Characterization, v. 60, n. 3, pp 165-172, 2009.

[6] KARLSSON, L., "Welding duplex stainless steels - A review of current recommendations", In: International Institute of Welding Conference, n. IX-2355-11, pp 1-17, 2011.

[7] BÖHLER, "GMAW solid wire, high alloyed, highly corrosion resistant", Technical Information, BÖHLER CN 25/9 CuT-IG, 2010.

[8] ALMENARA, M. D., VIDA, J., MATEO, A., et al., "Modelos empíricos para la predicción de la geometría del cordón en soldaduras a tope de un acero inoxidable dúplex 2205", Dyna, v. 78, n. 169, pp 206-215, 2011.

[9] ZAPPA, S., NOGERA, G., SVOBODA, H., et al., "Efecto del aporte térmico sobre la microestructura y la dureza en soldaduras de acero inoxidable dúplex”, In: CONAMET-SAM 2014, Argentina, 2014.

[10] KOTECKI, D. J., "Dilution control in single-wire stainless steel submerged arc cladding", Welding Journal, v.75, n. 2, pp 35s-45s, 1996.

[11] VITEC J. M., DAVIS, S. A. and HINMAN, C. R., "Improved ferrite number prediction model that accounts for cooling rate effects - Part 2: Model results", Welding Journal, v.82, n. 2, pp 43-s-50-s, 2003. 
[12] MARTINS, M., CASTELETTI, L. C., "Effect of heat treatment on the mechanical properties of ASTM A 890 Gr6A super duplex stainless steel”, Journal of ASTM International, v. 2, n. 1, pp 1-14, 2005. 